\title{
Personalidad y uso-abuso de éxtasis (MDMA)
}

\author{
Mª Paz García-Portilla, Pilar Alejandra Sálz, Begoña Paredes, Sonia Martínez, Julio Bobes
}

Area de Psiquiatría - Departamento de Medicina. Universidad de Oviedo

Enviar correspondencia: Ma Paz García-Portilla González. Area de Psiquiatría. Facultad de Medicina.

c/ Julián Clavería, 6 - 33006 Oviedo. Tfno: 98510 4219. Fax: 98510 3552. E-mail: albert@correo.uniovi.es

\begin{abstract}
Resumen
Objetivo: actualizar y sintetizar el conocimiento sobre la relación entre la personalidad y el uso-abuso de éxtasis. Método: se revisan y compilan los datos de la literatura científica sobre el tema. Resultados: los datos más sólidos apuntan hacia la presencia de rasgos de impulsividad, desinhibición y búsqueda de experiencias más prominentes en los consumidores de éxtasis que en los controles no consumidores. Estos datos de personalidad son consistentes con la hipofunción del sistema serotonérgico que los estudios neurobiológicos han puesto de manifiesto en esta población. Conclusión: se necesitan estudios prospectivos, doble ciego controlados con placebo, que permitan aclarar si los datos encontrados en los estudios naturalísticos son causa del consumo del éxtasis o por el contrario son consecuencia de dicho consumo.
\end{abstract}

Palabras claves: éxtasis, personalidad, impulsividad, búsqueda de sensaciones, serotonina

\begin{abstract}
Summary
Objective: to update and synthesize the knowledge about the relationship between personality and the use-abuse of ecstasy. Method: revision and collection of data from the scientific literature on the subject. Results: the strongest data point toward the presence of characteristics of impulsivity, disinhibition and seeking of experiences as being the most prominent in consumers of ecstasy than in non-consumer controls. These personality data are consistent with the hypofunction of the serotonergic system that neurobiological studies have demonstrated in this population. Conclusion: Prospective, double blind, placebocontrolled studies are necessary which permit us to clarify whether the data found in naturalistic studies are the cause of the consumption of ecstasy or whether they are the consequence of the consumption of such.
\end{abstract}

Key Words: ecstasy, personality, impulsivity, sensation seeking, serotonine

\section{INTRODUCCIÓN}

$\mathbf{L}$ a preocupación sobre la relación entre la personalidad y el uso-abuso de drogas existe ya desde hace varias décadas. Desde los ya clásicos estudios de Eysenck, buscando una relación entre el consumo de tabaco y la personalidad, han sido muchos los investigadores que han dedicado sus esfuerzos a arrojar alguna luz sobre el respecto (Sáiz y cols 2001). A pesar de ello, la interpretación de los resultados de los estudios sobre personalidad y consumo de drogas en general, y del éxtasis en particular, es una cuestión que todavía plantea importantes dificultades.

En primer lugar, los estudios epidemiológicos han demostrado que el perfil tipo del consumidor de sustancias es el policonsumo siendo excepcionales los casos de consumo puro, especialmente con el éxtasis, lo que implica dificultades a la hora de atribuir determinados efectos a una determinada sustancia en particular.

En segundo lugar, la mayor parte de los cuestionarios de personalidad están diseña- 
dos para evaluar rasgos estables de personalidad y no han demostrado, o no ha sido estudiada, su eficacia en la detección de cambios recientes.

Finalmente, la falta de estudios prospectivos hace que sea difícil determinar si los hallazgos deben ser interpretados como consecuencia del consumo, es decir, como cambios de personalidad inducidos por las drogas, o si por el contrario, esos rasgos de personalidad ya existían previamente y son los que predispusieron al sujeto al consumo. En suma se plantea la cuestión de si esos rasgos de personalidad que diferencian al consumidor del no consumidor son la causa o la consecuencia del consumo.

\section{ESTADO ACTUAL DEL TEMA}

Una cuestión de especial interés en la actualidad es poder determinar si los distintos rasgos de personalidad asociados al consumo de éxtasis son causa de dicho consumo o, si por el contrario, son su consecuencia. Así, existen estudios que han demostrado que como consecuencia de la exposición al éxtasis disminuiría la inhibición cognitiva de los comportamientos impulsivos, como sucede con otras drogas de abuso. Sin embargo, si se tienen en cuenta los hallazgos aportados por los estudios dirigidos a la búsqueda de un perfil de personalidad diferencial en los consumidores de drogas, cabría plantearse la cuestión en sentido contrario. Es decir, sería la falta de inhibición previa la que conduciría a un sujeto a consumir este tipo de sustancias. Esta cuestión tiene especial relevancia en el campo de la intervención (Calafat y cols 1985).

\section{PERSONALIDAD COMO CONSECUENCIA}

Estudios preclínicos han demostrado el efecto neurotóxico de la 3-4 metilendioximetanfetamina (MDMA) en los terminales sero- tonérgicos del cerebro de primates no humanos (Ricaurte y cols 1988) y posiblemente de los humanos (McCann y cols 1998, Semple y cols 1999). Aunque todavía no están bien establecidas las consecuencias funcionales de este daño (McCann y Ricaurte 1998), cada vez existe mayor evidencia, proveniente de estudios controlados, de un aumento de la impulsividad en los consumidores de MDMA como consecuencia del descenso de la actividad serotonérgica (Gerra y cols 1998, Morgan 1998, Parrot y cols 2000, Gerra y cols 2002).

Gerra y cols (1998, 2002), estudiando un grupo de 15 usuarios de MDMA que no habían utilizado durante un período prolongado de tiempo alcohol ni ninguna otra droga encontraron que, con respecto al grupo control, presentaban significativamente mayor búsqueda de sensaciones y hostilidad, demostrando además una correlación negativa entre ambos rasgos de personalidad y la respuesta de prolactina a la fenfluramina. En el estudio de Morgan (1998) el grupo de consumidores de MDMA (que también utilizaban otras sustancias ilegales) cometió significativamente más errores en el test de emparejamiento de figuras -MFF20-, una medida conductual de impulsividad, que los otros dos grupos de estudio (policonsumidores de sustancias que nunca utilizaron éxtasis y grupo control de no consumidores), sin que hubiese diferencias significativas en el nivel de ansiedad, depresión, hostilidad o funcionamiento cognitivo. Ampliando la muestra de estudio, el autor replica el hallazgo anterior que además se confirma al encontrar que también el grupo consumidor de MDMA obtenía puntuaciones significativamente más elevadas en el cuestionario de impulsividad, búsqueda de aventuras y empatía -IVE- que los otros dos grupos. Por su parte Parrot y cols (2000), en una muestra de población no clínica de jóvenes usuarios de éxtasis, encontró que los consumidores importantes de éxtasis (30-1000 ocasiones) obtenían puntuaciones significativamente más elevadas que los controles en impulsividad. Los consumidores "light" (1-20 ocasiones) se encontraban a medio camino entre los consumidores importantes y el 
grupo control de no consumidores de éxtasis.

\section{PERSONALIDAD COMO CAUSA}

Ya en 1983 Zuckerman asoció el consumo de drogas con rasgos de personalidad impulsivos; la desinhibición y la búsqueda de experiencias se relacionaba con la frecuencia del consumo de las distintas drogas, y esta relación era especialmente fuerte en el caso de los psicoestimulantes. Jaffe y Archer (1987) consideran que comparado con otros rasgos de personalidad, la búsqueda de sensaciones es el mejor predictor de consumo, y Luengo y cols (1996) identifican este rasgo como diferenciador entre adolescentes no-consumidores y aquellos que progresivamente van aumentando el consumo con el tiempo.

En nuestra comunidad Bobes y cols (2002) y Sáiz y cols (2001), han estudiado la asociación entre rasgos de personalidad y consumo de drogas en reclutas del Servicio Militar y en estudiantes de los institutos de Oviedo. En el estudio de Bobes y cols (2002) un total de 3643 reclutas cumplimentaron de forma válida los instrumentos de evaluación: el cuestionario de la OMS de consumo de alcohol y otras drogas (Smart y cols 1980), el cuestionario de personalidad de Eysenck -EPQ-A(Eysenck y Eysenck 1975) y la escala de búsqueda de sensaciones de Zuckerman -EBS(Zuckerman y cols 1978). El $10.9 \%$ de los reclutas afirmaron haber consumido MDMA alguna vez en la vida, el 7.8\% haberla consumido en el año previo, y el $4.5 \%$ en el mes previo. Los consumidores de MDMA se asociaban con mayores niveles de neuroticismo y dureza en el EPQ-A y con mayores puntuaciones en la escala de búsqueda de sensaciones y en sus cuatro subescalas que los no consumidores de drogas ilegales y que los consumidores de drogas ilegales diferentes de la MDMA.

Resultados similares se obtuvieron en el estudio de Sáiz y cols (2001) en el que se evaluó, con los mismos instrumentos que en el estudio anterior, una muestra de 2915 estudiantes de $2^{\circ}-4^{\circ}$ ESO y $1^{\circ}$ Bachiller en los centros públicos de Oviedo. De cara al análisis estadístico, dado que es prácticamente imposible localizar consumidores "puros" de MDMA, se dividió la muestra en tres subgrupos: grupo 1 (aquellos que sólo han consumido a lo largo de su vida drogas legales -tabaco y / o alcohol-), grupo 2 (aquellos que han consumido alguna vez drogas ilegales entre las que no figura la MDMA) y grupo 3 (aquellos que han consumido alguna vez MDMA, independientemente del resto de consumos legales e ilegales). Además se analizó si existía alguna diferencia en cuanto a personalidad entre los sujetos que tras haber consumido alguna vez en la vida siguieron consumiendo (presentaban consumo también en el último mes) y los que ya no consumían (no había consumos en el último mes). Para ello se creó una nueva variable, suma de consumo alguna vez en la vida y consumo en el último mes, en la que los sujetos se clasificaban en tres grupos: "abstinentes" (nunca consumieron MDMA, ni alguna vez, ni en el último mes); "experimentadores" (consumieron MDMA alguna vez, pero no en el último mes); y "reincidentes" (consumieron MDMA alguna vez y en el último mes). Por último, cabe señalar que estos análisis se realizarán de forma individualizada para cada sexo.

Las tablas I y || muestran los resultados obtenidos en el análisis de la asociación entre uso-abuso de sustancias alguna vez en la vida y el EPQ-A en varones y mujeres. Los resultados obtenidos en la dimensión neuroticismo ponen de manifiesto que los consumidores de sustancias ilegales diferentes de MDMA (grupo 2), de ambos sexos, son los que obtienen puntuaciones más elevadas en esta dimensión. No obstante, sólo se obtienen diferencias estadísticamente significativas en la submuestra de mujeres: grupo 1 vs grupo 2. En la dimensión intro-extroversión puede observarse que los consumidores de MDMA (grupo 3) obtienen puntuaciones más elevadas que los no consumidores de drogas ilegales. Finalmente, en la dimensión de psicoticismo en ambas submuestras de estu- 
Tabla I. Uso-abuso de MDMA alguna vez en la vida y EPQ-A / EBS (varones)

\begin{tabular}{|c|c|c|c|c|c|}
\hline & $\begin{array}{l}\text { Grupo } 1 \\
(n=839)\end{array}$ & $\begin{array}{l}\text { Grupo } 2 \\
(n=536)\end{array}$ & $\begin{array}{l}\text { Grupo } 3 \\
(n=73)\end{array}$ & $p^{*}$ & $\begin{array}{c}\text { Grupos } \\
\text { distintos }\end{array}$ \\
\hline \multicolumn{6}{|l|}{ EPQ-A } \\
\hline Neuroticismo & $12.29(4.77)$ & $13.04(5.14)$ & $12.81(5.56)$ & .023 & No diferencias \\
\hline Intro-extroversión & $14.53(3.56)$ & $15.54(2.98)$ & $15.49(2.69)$ & .000 & 1 vs 2,3 \\
\hline Psicoticismo & $4.76(2.91)$ & $6.22(3.37)$ & $8.47(3.88)$ & .000 & 1 vs 2 vs 3 \\
\hline \multicolumn{6}{|c|}{ Búsqueda Sensaciones } \\
\hline BEM & $7.49(2.44)$ & $7.78(2.09)$ & $8.19(1.92)$ & .009 & 1 vs 3 \\
\hline BEX & $4.54(1.49)$ & $5.97(1.82)$ & $6.89(1.76)$ & .000 & 1 vs 2 vs 3 \\
\hline DES & $6.10(1.98)$ & $7.88(1.69)$ & $8.38(1.49)$ & .000 & 1 vs 2 vs 3 \\
\hline SAP & $5.31(1.94)$ & $5.82(1.95)$ & $6.62(2.22)$ & .000 & 1 vs 2 vs 3 \\
\hline Total & $23.42(5.11)$ & $27.43(4.79)$ & $29.96(4.95)$ & .000 & 1 vs 2 vs 3 \\
\hline
\end{tabular}

\begin{tabular}{|c|c|c|c|c|c|}
\hline \multicolumn{6}{|c|}{ Tabla II. Uso-abuso de MDMA alguna vez en la vida y EPQ-A / EBS (mujeres) } \\
\hline & $\begin{array}{c}\text { Grupo } 1 \\
(\mathrm{n}=826) \\
\end{array}$ & $\begin{array}{c}\text { Grupo } 2 \\
(n=552) \\
\end{array}$ & $\begin{array}{c}\text { Grupo } 3 \\
(n=36)\end{array}$ & $p^{*}$ & $\begin{array}{l}\text { Grupos } \\
\text { distintos }\end{array}$ \\
\hline \multicolumn{6}{|l|}{ EPQ-A } \\
\hline Neuroticismo & $15.21(4.89)$ & $16.72(4.76)$ & $15.61(4.98)$ & .000 & 1 vs 2 \\
\hline Intro-extroversión & $14.26(3.60)$ & $15.24(3.32)$ & $15.81(2.86)$ & .000 & 1 vs 3 \\
\hline Psicoticismo & $3.32(2.25)$ & $4.53(2.82)$ & $5.47(3.52)$ & .000 & 1 vs 2 vs 3 \\
\hline \multicolumn{6}{|c|}{ Búsqueda Sensaciones } \\
\hline BEM & $6.94(2.51)$ & $7.35(2.25)$ & $7.78(1.88)$ & .003 & 1 vs 3 \\
\hline BEX & $5.12(1.50)$ & $6.62(1.61)$ & $7.64(1.55)$ & .000 & 1 vs 2 vs 3 \\
\hline DES & $4.98(2.12)$ & $6.86(1.90)$ & $7.55(1.44)$ & .000 & 1 vs 2 vs 3 \\
\hline SAP & $4.99(3.47)$ & $5.69(1.79)$ & $5.97(1.92)$ & .000 & 1 vs 3 \\
\hline Total & $21.97(5.13)$ & $26.51(4.63)$ & $29.00(3.94)$ & .000 & 1 vs 2 vs 3 \\
\hline
\end{tabular}

diantes se observa que los tres grupos son diferentes entre sí, de tal manera que se puede hablar de un gradiente de psicoticismo en función del consumo: a más sustancias mayor puntuación en psicoticismo. Desde un punto de vista clínico, es necesario señalar que tan sólo la dimensión de psicoticismo adquiere trascendencia clínica, ya que es la única en la que los consumidores de sustancias ilegales (con independencia de que se incluya o no la MDMA), de ambos sexos, se sitúan en rangos iguales o superiores al percentil 85.

Con respecto a la búsqueda de sensaciones puede decirse, en conjunto, que tanto en hombres como en mujeres las puntuaciones aumentan significativamente en función del consumo, de modo que son significativamente más bajas en los grupos que no consumen sustancias ilegales y más elevadas en el grupo de jóvenes que consumen MDMA (generalmente, junto a otras drogas ilegales) (tablas I y II). Desde el punto de vista clínico, este instrumento se comporta de modo muy similar al anterior, mayor desviación respecto a la norma en función de la importancia del consumo. No obstante, la trascendencia clínica es escasa: únicamente en la escala de desinhibición (los grupos 2 y 3) y en la puntuación total (grupo 3), la muestra de estudiantes mujeres se diferencia de la población de referencia (mujeres estudiantes españolas -Pérez y Torrubia, 1986-) 2 ó más desviaciones estándar. 


\begin{tabular}{|c|c|c|c|c|c|}
\hline & $\begin{array}{l}\text { Abstinentes } \\
(n=1375)\end{array}$ & $\begin{array}{c}\text { Experimentadores } \\
\qquad(n=38)\end{array}$ & $\begin{array}{c}\text { Reincidentes } \\
\quad(n=35)\end{array}$ & $p^{*}$ & $\begin{array}{c}\text { Grupos } \\
\text { distintos }\end{array}$ \\
\hline \multicolumn{6}{|l|}{ EPQ-A } \\
\hline Neuroticismo & $12.58(4.93)$ & $12.80(5.67)$ & $12.57(5.62)$ & NS & No diferencias \\
\hline Intro-extroversión & $14.93(3.39)$ & $15.87(2.49)$ & $14.91(2.85)$ & NS & No diferencias \\
\hline Psicoticismo & $5.33(3.18)$ & $7.20(3.13)$ & $9.69(4.26)$ & .000 & 1 vs 2 vs 3 \\
\hline \multicolumn{6}{|c|}{ Búsqueda Sensaciones } \\
\hline BEM & $7.60(2.31)$ & $8.07(2.16)$ & $8.14(1.88)$ & NS & No diferencias \\
\hline BEX & $5.09(1.77)$ & $7.32(1.61)$ & $6.40(1.79)$ & .000 & 1 vs 2 vs 3 \\
\hline DES & $6.79(2.06)$ & $8.25(1.43)$ & $8.43(1.70)$ & .000 & 1 vs 2 y 3 \\
\hline SAP & $5.51(1.96)$ & $6.72(2.34)$ & $6.29(2.20)$ & .000 & 1 vs 2 y 3 \\
\hline Total & $24.98(5.35)$ & $30.15(4.75)$ & $29.29(5.67)$ & .000 & 1 vs 2 y 3 \\
\hline
\end{tabular}

\begin{tabular}{|c|c|c|c|c|c|}
\hline \multicolumn{6}{|c|}{$\begin{array}{l}\text { Tabla IV: Diferencias en el perfil EPQ-A y EBS entre los que nunca consumieron MDMA (absti } \\
\text { nentes), los que probaron pero no continuaron (experimentadores), y los que habiendo proba- } \\
\text { do continuaron consumiendo (reincidentes) (mujeres) }\end{array}$} \\
\hline & $\begin{array}{l}\text { Abstinentes } \\
(n=1378)\end{array}$ & $\begin{array}{l}\text { Experimentadores } \\
\qquad(\mathrm{n}=24)\end{array}$ & $\begin{array}{l}\text { Reincidentes } \\
\quad(n=12)\end{array}$ & $p^{*}$ & $\begin{array}{l}\text { Grupos } \\
\text { distintos }\end{array}$ \\
\hline \multicolumn{6}{|l|}{ EPQ-A } \\
\hline Neuroticismo & $15.81(4.89)$ & $15.04(5.43)$ & $17.17(3.61)$ & NS & No diferencias \\
\hline Intro-extroversión & 14.65 (3.52) & $15.40(3.03)$ & $16.67(2.19)$ & NS & No diferencias \\
\hline Psicoticismo & $3.80(2.56)$ & $5.52(3.50)$ & $5.33(3.55)$ & .001 & 1 vs 2 y 3 \\
\hline \multicolumn{6}{|c|}{ Búsqueda Sensaciones } \\
\hline BEM & $7.11(2.42)$ & $7.48(2.06)$ & $8.58(1.16)$ & NS & 1 vs 3 \\
\hline BEX & $5.72(1.71)$ & $7.48(1.64)$ & $7.92(1.31)$ & .000 & 1 vs 2 y 3 \\
\hline DES & $5.73(2.23)$ & $7.56(1.45)$ & $7.67(1.50)$ & .000 & 1 vs 2 y 3 \\
\hline SAP & $5.27(2.94)$ & $6.00(1.53)$ & $6.25(2.83)$ & NS & No diferencias \\
\hline Total & $23.77(5.41)$ & $28.52(4.13)$ & $30.58(3.65)$ & .000 & 1 vs 2 y 3 \\
\hline
\end{tabular}

Cuando evaluamos los rasgos de personalidad en función de la persistencia del consumo -abstinentes, experimentadores y reincidentes- (tablas III y IV) no se obtuvieron diferencias en las dimensiones neuroticismo y intro-extroversión. Sin embargo, en la dimensión psicoticismo se aprecia, en el caso de los hombres, un claro incremento de la puntuación obtenida en función del nivel de consumo de MDMA y, en el caso de las mujeres, sólo se observan diferencias entre el hecho de haber consumido (experimentadores y reincidentes) o no (abstinentes)
MDMA. Además esta diferencia estadística tiene también significado clínico; en ambas muestras se observa que experimentadores y reincidentes se sitúan en percentiles clínicamente significativos (superiores al percentil 85) y, los hombres abstinentes están en el percentil 85.

Respecto a la búsqueda de sensaciones en la muestra de hombres se observa, que en la subescala de búsqueda de excitación, las mayores puntuaciones son obtenidas por el grupo de experimentadores, seguido del grupo de reincidentes y, por último, de los 
abstinentes, mientras que en las subescalas desinhibición, susceptibilidad al aburrimiento y puntuación total sólo se observan diferencias entre abstinentes y consumidores (independientemente de que sean experimentadores o reincidentes) (tabla III). En el caso de las mujeres (tabla IV), el panorama varía ligeramente: diferencias entre abstinentes y consumidores en las subescalas búsqueda de excitación, desinhibición y puntuación total, diferencias entre abstinentes y reincidentes en la subescala búsqueda de emoción y aventura. Desde el punto de vista clínico, de nuevo son la subescala desinhibición y la puntuación total, en el sexo femenino, las que mayores desviaciones presentan respecto a la norma, de modo que las estudiantes experimentadoras y reincidentes superan las 2 desviaciones estándar por encima de la norma (Pérez y Torrubia, 1986), en dichas subescalas.

\section{CONCLUSIONES}

El uso-abuso de MDMA parece asociarse a rasgos prominentes de impulsividad y búsqueda de sensaciones, sin que hasta el momento se pueda determinar si ello es causa o consecuencia del consumo. Por otra parte, el hecho de que prácticamente no existan consumidores puros de MDMA dificulta la atribución exclusiva de dichos rasgos al éxtasis. Por tanto, se necesitan estudios adicionales prospectivos, doble ciego controlados con placebo que ayuden a clarificar los hallazgos actuales.

\section{REFERENCIAS}

Bobes J, Sáiz PA, González MP, Bascarán MT, Bousoño M, Ricaurte GA, McCann UD. Use of MDMA and other illicit durgas by young adult males in northern Spain. Eur Addict Res 2002; 8: 147-154.
Calafat A, Amengual M, Farres C, Palmer A. Estilo de vida y hábitos de consumo de drogas entre estudiantes de enseñanza media. Boletín de Estupefacientes 1985; XXXVII (2-3): 121-131.

Eysenck HJ, Eysenck SBG. Eysenck personality questionnaire-junior (EPQ-J) \& adult (EPQ-A). London: Hodder and Stoughton Educational. 1975.

Gerra G, Zaimovic A, Giucastro G, Maestri D, Monica $C$, Sartori R, et al. Serotonergic function afeter (+/-)3,4-methylene-dioxymethamphetamine (Ectasy) in humans. Int Clin Psychopharmacol 1998; 13: 1-9.

Gerra G, Zaimovic A, Moi G, Giusti F, Gardini S, Delsignore R, Laviola G, Macchia T, Brambilla F. Effects of (+/-) 3,4-methylene-dioxymethamphetamine (ecstasy) on dopamine system function in humans. Behav Brain Res 2002;134(1-2): 403-410.

Jaffe LT, Archer RP. The prediction of drug use among college students from MMPI, MCMI and sensation seeking scales. Journal of Personality Assessment 1987; 51: 243-253.

Luengo A, Otero-López JM, Romero E, Gómez JA. Efectos de la necesidad de búsqueda de sensaciones sobre la involucración en el consumo de drogas de los adolescentes. Análisis y Modificación de la Conducta 1996; 22 (86): 683-708.

McCann U, Szabo Z, Scheffel U, Dannals RF, Ricaurte GA. Positron emission tomographic evidence of toxic effect of MDMA (Ectasy) on brain serotonin neurons in human beings. Lancet 1998; 352: 1433-1437.

McCann UD, Ricaurte GA. Aproximación a la neurobiología y neurotoxicidad comparada inducida por la MDMA. En: J Bobes, P Lorenzo, PA Sáiz (eds.). Éxtasis (MDMA): un abordaje comprehensivo. Barcelona: Masson. 1998, pp: 89-97.

Morgan MJ. Recreational use of "ecstasy" (MDMA) is associated with elevated impulsivity. Neuropsychopharmacology 1998; 19(4): 252-64. Parrott AC, Sisk E, Turner JJ. Psychobiological problems in heavy 'ecstasy' (MDMA) polydrug users. Drug Alcohol Depend 2000; 60(1):105-10.

Pérez J, Torrubia R. Fiabililidad y validez de la versión española de la Escala de Búsqueda de Sensaciones (Forma V). Revista Latinoamericana de Psicología 1986; 18: 7-22.

Ricaurte GA, Delanney LE, Wiener SG, Irwin I, Langston JW. 5-Hydroxyindoleacetic acid in 
cerebrospinal fluid reflects serotonergic damage induced by 3,4-methylenedioxymethamphetamine in CNS of non-human primates. Brain Res 1988; 474: 359-363.

Sáiz PA, González MP, Paredes B, Delgado JM, López JL, Martínez S, Bobes J. Consumo de MDMA (éxtasis) en estudiantes de secundaria. Adicciones 2001; 13(2): 159-171.

Sáiz PA, González MP, Paredes B, Martínez S, Delgado JM. Personalidad y uso-abuso de cocaína. Adicciones 2001; 13(supl 2): 47-59.

Semple DM, Ebmeier KP, Glabus MF, O'Carroll RE, Johnstone EC. Reduced in vivo binding to the serotonin transporter in the cerebral cortex of MDMA (Ectasy) users. Br J Psychiatry 1999; 175: 63-69.
Smart RG, Hughes PH, Johnston LD, Anumonye A, Khant U, Medina ME, Navaratnam V, Poshyachinda V, Varma VK, Wadud KA. Méthodologie pour des enquètes sur I'usage des drogues chez les étudiants. Publication offset $N^{\circ} 50$. Genève: OMS. 1980.

Zuckerman M. A biological theory of sensation-seeking. En: Zuckerman M (ed.). Biological bases of sensation-seeking, impulsivity, and anxiety. Hillsdale: Erlbaum. 1983, pp. 37-76.

Zuckerman M, Eysenck SBG, Eysenck HJ: Sensation-seeking in England and America: Crosscultural, age and sex comparisons. J Consulting Clinical Psychology 1978; 46: 139-149. 
